\title{
Responsive Politikgestaltung in den deutschen Bundesländern? Versuch einer Systematisierung und Konzeption eines Forschungsprogramms
}

\author{
Georg Wenzelburger $(\mathbb{D} \cdot$ Stefan Wurster (D) Markus B. Siewert (D)
}

Online publiziert: 20. Mai 2020

(C) Der/die Autor(en) 2020

Zusammenfassung Die politikwissenschaftliche Literatur zum deutschen Föderalismus ist überaus vielfältig. Neben Analysen der institutionellen Arrangements, ihrer Veränderungen sowie der Dynamiken des deutschen Verbundföderalismus, finden sich auch zahlreiche Untersuchungen zu einzelnen Politikfeldern, die sowohl die Interaktionen zwischen Bund und Ländern als auch die Varianz zwischen den Policies der Länder samt ihrer Bestimmungsfaktoren untersuchen. Darüber hinaus haben sich in den vergangenen Jahrzehnten eigene Forschungszweige zu Parteien im Bundesstaat und zur Parlamentsforschung auf Länderebene etabliert. Trotz dieser großen Forschungsaktivität sind jedoch einige zentrale Fragen der Politikwissenschaft zum Zusammenspiel zwischen Wählern, Parteien, Parlamenten und Regierungen sowie deren Wirkung auf politischen Outputs und Outcomes weiterhin unbeantwortet. Dies ist, so das Argument dieses Beitrags, insbesondere der fehlenden Zusammenführung einzelner Literaturstränge und der noch unzureichenden empirischen Datenbasis geschuldet. Mittels einer Systematisierung des gegenwärtigen Literaturstands entwirft der Aufsatz ein Forschungsprogramm, das auf eine umfassende Analyse des politischen Willensbildungs- und Entscheidungsfindungsprozesses in den deutschen Bundesländern abstellt und Fragen der Responsivität und Rückkopplung systematisch in den Blick nimmt.

\footnotetext{
G. Wenzelburger $(\bowtie)$

Fachbereich Sozialwissenschaften, Technische Universität Kaiserslautern, Kaiserslautern, Deutschland

E-Mail: georg.wenzelburger@sowi.uni-kl.de
}

S. Wurster · M. B. Siewert

Hochschule für Politik München, München, Deutschland

S. Wurster

E-Mail: stefan.wurster@hfp.tum.de

M. B. Siewert

E-Mail: markus.siewert@hfp.tum.de 
Schlüsselwörter Responsivität · Bundesländervergleich · Agenda-Setzung · politische Willensbildung · politischer Entscheidungsprozess · Policyoutputs und -Outcomes · Rückkopplung · Forschungsprogramm · empirische Datengrundlage

\title{
Responsive policy-making in the German federal states? An attempt to systematize and to develop a research program
}

\begin{abstract}
The political science literature on German federalism is extremely diverse. Many scholars have analyzed the institutional arrangements, their transformations, and the dynamics of the German federal state. Another strand of literature focuses on individual policy fields and examines both the interactions between the federal and the state level as well as the variance between the Länder policies and its determinants. Moreover, there has been vibrant research on political parties and parliamentary practices at the Länder level over the last decades. Yet despite this great research activity central questions regarding the interaction between voters, parties, parliaments and governments and their effects on political outputs and outcomes are still unaddressed. According to the argument in this article, this is particularly due to the missing linkages between the individual strands of the literature and the lack of a database which would allow for integrated empirical analyses. Building on a systematization of the literature, this essay lays out a research program that is based on a comprehensive analysis of the political opinion-formation and decisionmaking process in the German federal states and systematically focuses on questions of responsiveness and feedback.
\end{abstract}

Keywords Responsiveness - Subnational comparison - Opinion-formation · Agenda-setting · Decision-making · Policy outputs and policy outcomes · Feedback $\cdot$ Research program $\cdot$ Empirical database

\section{Einführung}

Studien zur politischen Willensbildung und Entscheidungsfindung in föderalen Systemen sind ein klassischer Forschungstopos der Politikwissenschaft, insbesondere in Deutschland. Dabei lässt sich der umfangreiche Forschungsstand zum föderalen System der Bundesrepublik in zwei große Strömungen einteilen. Ein erster Literaturstrang beschäftigt sich mit den Beziehungen zwischen Bund und Ländern sowie der Koordination zwischen den Ländern im verbundföderalistischen System der Bundesrepublik. Hierbei stehen insbesondere Fragen der Politikverflechtung (Scharpf et al. 1976; Scharpf 1985), der mangelnden Passung von Parteienwettbewerb und Verhandlungszwang (Lehmbruch 1976) sowie Dynamiken der Kooperation zwischen den Akteuren (Benz 1985; Kropp 2010; Hegele und Behnke 2013) und (De)Zentralisierungstendenzen (Kaiser und Vogel 2017) im Zentrum der Analyse (zur internationalen Literatur, siehe Bolleyer 2014). Ein zweiter Literaturstrang nimmt die Länderebene vergleichend in den Blick und untersucht Gemeinsamkeiten und Unterschiede der Politik der Bundesländer. Diese Arbeiten richten ihr Interesse u. a. auf Parteien und Parteiensysteme der Länder (Jun et al. 2008; Bräuninger und 
Debus 2012; Bräuninger et al. 2020), Landtagswahlen und -wahlkämpfe (Völkl et al. 2008; Rauh 2016), Aussagen zu und Formierung von Koalitionen auf Landesebene (Spier 2013; Best 2015; Baumann et al. 2017) sowie institutionelle Unterschiede zwischen den politischen Systemen der Länder (Leunig 2007; Freitag und Vatter 2008). Zudem lässt sich feststellen, dass die Forschungsaktivitäten zur Varianz zwischen den deutschen Ländern mit Blick auf Politikinhalte (Schmidt 1980; Hildebrandt und Wolf 2008, 2016; Wagschal und Wenzelburger 2009; Hörisch und Wurster 2017, 2019) sowie Politikergebnisse im Sinne von Outputs und Outcomes (Wurster 2017; Wurster und Hagemann 2018, 2019) deutlich zugenommen haben (siehe insbesondere auch die beiden Aufsatzsammlungen von Jeffery et al. 2016; und Sack und Töller 2018).

Wir widmen uns im vorliegenden Literaturbericht zuvorderst letzterer Perspektive und blicken auf die vergleichende Bundesländerforschung - wenngleich föderale Dynamiken selbstverständlich immer eine Rolle spielen. Vergleicht man hier den Stand der Forschung insbesondere mit neueren US-amerikanischen Debatten, so zeigt sich, dass sich diese in den letzten Jahren verstärkt den ,großen Fragen “ zu Repräsentation oder Responsivität (etwa Lax und Phillips 2012; Pacheco 2013) zugewandt haben, während für Deutschland dazu bisher kaum systematisch-empirische Forschungen vorliegen. Dabei ist etwa die Frage, ob ,der demokratische Prozess die Regierung dazu bewegt, diejenige Politik zu machen und zu implementieren, welche die Bürger*innen wollen“ (Powell 2004, S. 91; eigene Übersetzung) und sich damit responsiv zu zeigen, auch auf Länderebene von zentraler demokratietheoretischer und -praktischer Bedeutung. Angesichts dieser Forschungslücke stellen wir in diesem Literaturbericht die folgenden drei Fragen: Erstens, welche Resultate hat die bisherige Forschung in Bezug auf einzelne Aspekte von Repräsentation und Responsivität hervorgebracht? Zweitens, welche Schlussfolgerungen ergeben sich hieraus mit Blick auf Responsivität im deutschen Ländervergleich? Und drittens, wie könnte eine Forschungsagenda aussehen, die zentrale Leerstellen adressiert?

Der Beitrag gliedert sich wie folgt: In Abschnitt 2 stellen wir zunächst die Kette der Responsivität auf theoretischer Ebene vor, bevor Abschnitt 3 einen Überblick zum Forschungsstand in Bezug auf die einzelnen Elemente dieser Kette im deutschen Föderalstaat gibt. In Abschnitt 4 skizzieren wir schließlich ein zukünftiges Forschungsprogramm, welches Aspekte von Responsivität in den deutschen Bundesländern (noch) stärker als bisher in den Blick nimmt.

\section{Die Kette der Responsivität}

Ob und gegebenenfalls wie sich die Präferenzen einzelner Bürger*innen in tatsächlichen Politikinhalten wiederspiegeln, ist eine alte politikwissenschaftliche Frage. Sie fußt auf Analysen zur Repräsentation (Przeworski et al. 1999), die vor allem in der US-amerikanischen Politikwissenschaft eine lange Tradition aufweisen (Miller und Stokes 1963; Pitkin 1967; Page und Shapiro 1983). Aufbauend auf der Studie von Powell (2004), lässt sich dabei eine demokratische Kette der Responsivität (chain of responsiveness) konzipieren, die der Theorie nach die Präferenzen der Wählerschaft (Input) über die Agendasetzung in Regierungspolitik (Throughput) und somit in Out- 
puts und Outcomes übersetzt (opinion-policy-linkage; ähnlich auch Esaiasson und Wlezien 2017). Dabei besteht der größte Unterschied zwischen den ursprünglichen Überlegungen von Powell und unseren folgenden Ausführungen in der expliziten Modellierung von politischen Parteien, und deren Partei- und Wahlprogrammen, als Transmissionsriemen - was angesichts der zentralen Rolle politischer Parteien im deutschen System sinnvoll erscheint (dazu auch: Dalton et al. 2011). Entsprechend der Annahmen des thermostatic models (Soroka und Wlezien 2010) sollte darüber hinaus eine Feedback-Schleife bestehen, welche idealiter die Regierungsperformanz (Outputs und Outcomes) rückbindet an die Forderungen und Präferenzen der Bürger*innen.

Angesichts der umfangreichen international-vergleichenden Forschungsaktivität ist es überraschend, dass die Frage nach der opinion-policy-linkage und ihrem Zustandekommen auf subnationaler Ebene nur selten untersucht wird - zumal Gliedstaaten erhebliche Kompetenzen in zahlreichen Politikfeldern besitzen. Erst in den letzten Jahren sind Studien zu den US-Einzelstaaten entstanden, die zeigen, dass unter bestimmten Bedingungen - Formen von dynamischer Responsivität sowie von Kongruenz feststellbar sind (Lax und Phillips 2012; Pacheco 2013; Caughey und Warshaw 2017; Simonovits et al. 2019). Für die subnationale Ebene in Deutschland fehlen solche Analysen, die das Zusammenspiel von Bürgerpräferenzen und politischen Willensbildungs- und Entscheidungsfindungsprozess systematisch und umfassend in den Blick nehmen, dagegen weitgehend. Vor diesem Hintergrund gibt der nächste Abschnitt einen Überblick über die vorhandenen empirischen Befunde zu einzelnen Elementen der Responsivitätskette in den deutschen Ländern und zeigt bestehende Leerstellen auf.

\section{Forschungsstand und bestehende Desiderata}

\subsection{Präferenzen der Wählerschaft und Agendasetzung auf Landesebene}

Die Präferenzen der Wählerschaft und die Problemdefinition stehen am Anfang des politischen Prozesses und sind maßgeblich dafür, ob ein bestimmtes Thema überhaupt als ein politisches Problem angesehen wird und damit die Möglichkeit erhält, auf die Regierungsagenda zu gelangen (Kingdon 1984, S. 23-27; Jones 1994). Powell (2004, S. 92) beschreibt diesen Prozess auch als ,structuring choices“. Wann ein Thema in der Öffentlichkeit Aufmerksamkeit erreicht und zu einem politischen Problem wird, hängt dabei von vielen unterschiedlichen Faktoren ab, z. B. vom Verhalten politischer und gesellschaftlicher Akteure, von spezifischen Ereignissen oder der medialen Aufmerksamkeit. Für die deutschen Länder wurden die landesspezifischen Einflussfaktoren der Problemdefinition bisher kaum analysiert. Dabei ist durchaus zu vermuten, dass spezifische Elemente der Problemdefinition auf Landesebene insbesondere dann eine Rolle spielen, wenn die Problemlösung in die Kompetenzbereiche der Landesregierungen fallen (z. B. bei der Bildungspolitik) oder wenn das Thema einen starken regionalen Bezug hat. Dafür spricht etwa die Medienanalyse von Tenscher und Schmid (2009, S. 69-70), die für drei ausgewählte Landtagswahlen zeigt, dass regionale Medien überproportional diejenigen Fragen thematisieren, 
welche die Kompetenzen der Länder betreffen. Im Gegensatz dazu erwartet von Blumenthal (2010, S. 191), dass bereits bei der Problemdefinition ,,angesichts einer weitgehend bundesweit orientierten politischen Öffentlichkeit möglicherweise die Weichen für eine einheitliche Herangehensweise gestellt" werden.

Einen weiteren Ansatzpunkt in diesem Zusammenhang bilden Studien zu sogenannten Nebenwahlen (second-order elections) in föderalen Systemen (Müller und Debus 2012). Diese Arbeiten geben Hinweise darauf, ob bundespolitische oder landespolitische Themen bei einer Wahl besonders relevant sind. Hier finden etwa Krumpal und Rauhut (2006, S. 12), dass insbesondere westdeutsche Wähler*innen die Landespolitik bei Landtagswahlen als wichtiger für ihre Wahlentscheidung erachten als die Bundespolitik. Völkls (2008, S. 163) Untersuchung verdeutlicht darüber hinaus, dass Wähler*innen durchaus zwischen Landes- und Bundesthemen unterscheiden können. Allerdings spielen auch allgemeine Sachfragen, wie etwa die Arbeitslosenquote, für Landtagswahlen eine wichtige Rolle, sodass sowohl Verdrängungseffekte als auch Kontaminationen sowie eine gegenseitige Beeinflussung von Themenwichtigkeiten zu erwarten sind. Zu ähnlichen Ergebnissen kommen Untersuchungen in anderen Föderalstaaten. So zeigen etwa die Ergebnisse von Cabeza (2018) zu spanischen Regionalwahlen, dass Wähler*innen abhängig vom Kontext (z.B. wirtschaftliche Situation) eher regionale oder eher nationale Themen als wichtig für ihre Wahlentscheidung erachten.

Die meisten empirischen Studien zur Responsivität nutzen Daten zu themenspezifischen Einstellungen der Bürger*innen - etwa die Frage, welche Issues sie für wichtig erachten -, um die öffentliche Agenda zu definieren (dazu kritisch Wlezien 2016). Angesichts der zentralen Rolle von Präferenzen als Ausgangs- und Endpunkt der Kette der Responsivität sind Informationen zu den Präferenzen daher von großer Bedeutung. Abgesehen von einzelnen, allerdings nur in großen zeitlichen Abständen stattfindenden Landtagswahlstudien, fehlen auf Bundesländereben Analysen, welche die Dynamiken einzelner Themenkomplexe über längere Zeiträume hinweg präzise darstellen. Bisher konnten nur zu einzelnen besonders bedeutsamen Themenkomplexen, wie beispielsweis der Flüchtlingskrise im Jahr 2016 (Debus 2017; Kortmann und Stecker 2019), entsprechende Analysen durchgeführt werden.

Über spezifische Einstellungen hinaus hat auch das politische Wissen der Wähler*innen einen Einfluss auf die Agendasetzung auf Landesebene. Können Bürger*innen nämlich tatsächlich bewerten, dass ihre Landesregierung bestimmte Kompetenzen besitzt und demnach für gewisse Politiken verantwortlich ist, erhöht sich die Chance, dass dieses Thema potentiell als ein landespolitisches Problem definiert wird. Entsprechend wirkt politisches Wissen auch Strategien der Schuldvermeidung (blame avoidance) entgegen, wenn Landesregierungen für schlechte Performanz auf die Bundesebene verweisen (Cabeza et al. 2016; Stecker 2015). Inwiefern solches Wissen bei Bürger*innen auf Landesebene vorhanden ist, wurde bisher jedoch empirisch nur sehr eingeschränkt untersucht (ein internationaler Vergleich mit zwei deutschen Ländern findet sich bei Golder et al. 2017, S. 119-124). 


\subsection{Politikformulierung durch Parteien auf Landesebene}

Wenn ein Thema als politisches Problem definiert und auf die öffentliche Agenda gelangt ist, besteht der nächste Schritt in seiner Übersetzung in politische Programme und über Wahlen sowie mittels Regierungsbeteiligung politischer Parteien in Public Policies. In den vergangenen Jahrzehnten wurden enorme Fortschritte in der Vermessung dieser Übersetzungsleistung durch politische Parteien erzielt. So ist es zum Beispiel möglich, über die Daten des Comparative Manifesto Projects die Betonung einzelner Themen in Parteiprogrammen für eine große Zahl von Parteien über Zeit nachzuvollziehen (Volkens et al. 2018). Alternativ wurde über Expertenbefragungen erhoben, welche Themen von politischen Parteien als wichtig erachtet werden (Laver und Hunt 1992; Benoit und Laver 2006; Polk et al. 2017). Schließlich ist es möglich, die Positionierung von Parteien und die Betonung bestimmter Themen mittels textanalytischer Verfahren zu erheben und somit nicht nur Dynamiken des Parteienwettbewerbs nachzuzeichnen (Adams et al. 2005), sondern auch zu überprüfen, ob und in welchem Ausmaß Parteien tatsächlich das auf Basis ihrer Programme erhaltene Wählermandat in politische Outputs überführen (Klingemann et al. 1994; Budge 2015). Für diese policy-to-program linkages spricht u. a. das Vorliegen eines empirischen Zusammenhangs zwischen der Betonung bestimmter Themen im Wahlprogramm und politischen Outputs (siehe etwa Wenzelburger et al. 2019).

Trotz der vergleichsweise hohen und innovativen Forschungstätigkeit in Bezug auf die Analyse von Parteiprogrammen und deren Einfluss auf Policies (u. a. Bräuninger und Debus 2012; Bräuninger et al. 2020; Wenzelburger 2013; Bräuninger und Giger 2016; Hörisch und Wurster 2017; Bräuninger et al. 2019) besteht auf Länderebene eine Forschungslücke bei der themenspezifischen Positionierung der Landesparteien. Im Gegensatz zu einigen anderen europäischen Staaten, in denen die Programme der regionalen Parteien im Rahmen des Regional Manifesto Project erfasst wurden (Alonso et al. 2013), ist für Deutschland bis dato nicht erhoben worden, welche thematischen Schwerpunkte Parteien in ihren Landtagswahlprogrammen setzen und mit welchen konkreten Politiken sie sich den Wählern präsentieren. Zwar haben immer wieder einzelne Forscher versucht, mittels textanalytischer Verfahren eine themenspezifische Vermessung der Parteipositionen zu erreichen (Seher und Pappi 2011; Bräuninger und Debus 2012; Bräuninger et al. 2020; Ewert et al. 2018). Dennoch gibt es immer noch viele Lücken in Bezug auf eine übergreifende und in sich systematische Aufbereitung des Politikangebots von Parteien auf Landesebene. Mit Blick auf die Analyse von Responsivität ist diese Leerstelle jedoch problematisch, weil theoretisch von Issue-spezifischer Responsivität ausgegangen wird. Zwar ist es prinzipiell möglich, die Reaktion von Parteien auf Wählerpräferenzen auch auf aggregiertem Niveau (z.B. auf einer übergreifenden Links-Rechts-Dimension) zu analysieren (Bräuninger und Debus 2012, S. 187-201). Allerdings bleiben hier die eigentlichen Dynamiken auf der Ebene der Sachthemen im Dunkeln, wobei gerade diese Responsivität bei Sachfragen zumindest auf Ebene der Nationalstaaten relevant zu sein scheint.

Wie für die Agendasetzung gilt im Übrigen auch für die Politikformulierung von Parteien auf Landesebene, dass diese nicht isoliert von der Bundespolitik betrachtet werden kann. Dies betrifft sowohl die Frage, welche Themen in Parteiprogrammen 
stärker oder weniger stark betont werden, als auch die in den Landtagswahlprogrammen formulierten Politiken. Denn der Forschungsstand zu den Inhalten regionaler Parteiprogramme zeigt, dass Parteien nicht nur diejenigen Policies in ihren Programmen aufnehmen, welche tatsächlich auf Landesebene entschieden werden können, sondern strategisch absichtsvoll auch nationale Themen in Wahlprogrammen betonen (Alonso et al. 2013; Cabeza et al. 2016; Golder et al. 2017). Diese Mischungsverhältnisse wurden bisher vor allem im Kontext des Regional Manifestos Project für Spanien und Großbritannien untersucht. Auch für Deutschland zeigen Analysen, dass Landesparteien ihre Positionen sowohl in Abhängigkeit von der allgemeinen Parteilinie als auch in Bezug auf regionale Elektorate verändern (Müller 2013). Daneben findet Stecker (2015), dass Landesparteien sich im Parteienwettbewerb strategisch zwischen bundespolitischen und landespolitischen Themen positionieren. Zudem weist eine explorative Auswertung auf lokaler Ebene im Zuge des Local Manifesto Projects darauf hin, dass die Betonung nationaler Themen in den Programmen von Parteien in großen deutschen Städten durchaus variiert (Gross und Jankowski 2019). Schließlich finden qualitative Untersuchungen zu einzelnen Wahlen und Wahlkämpfen ebenfalls jeweils spezifische Mischungsverhältnisse von bundes- und landespolitischen Themen (siehe etwa die Beiträge in Tenscher und Rußmann 2016).

\subsection{Politische Outputs und Outcomes auf Landesebene}

Eine Untersuchung der Responsivitätskette ist ohne den Einbezug von politischen Entscheidungsprozessen (decision-making) und deren konkreter Ergebnisse in Form von Policy-Outputs/-Outcomes unvollständig. Vergleichende Studien zum PolicyOutput haben dabei in der Vergangenheit vor allem auf finanzielle Messgrößen (Staatsfinanzen) und Gesetzgebungsindikatoren zurückgegriffen. Staatsausgaben finden insbesondere in der Forschungsrichtung zum thermostatic model ihre Anwendung (Wlezien und Soroka 2012; Soroka und Wlezien 2015), wobei in der Regel deren Entwicklung mit den Veränderungen der öffentlichen Meinung abgeglichen wird. Angesichts der Kritik gegenüber Staatsausgaben als angemessenem Proxy für die Staatstätigkeit (Green-Pedersen 2004; Clasen und Siegel 2007), wurde in den vergangenen Jahren vermehrt die Gesetzgebung als direktester Policy-Output erfasst. Hierbei sind eine Reihe an Projekten entstanden, wie etwa das Comparative Policy Agendas Project (Baumgartner und Jones 1991; Baumgartner et al. 2006), das Consensus-Project (Bauer und Knill 2014) oder Arbeiten zu einzelnen Politikfeldern (Jensen und Wenzelburger 2020; Wenzelburger 2020). Dabei wurde die Kongruenz zwischen Präferenzen der Bevölkerung einerseits und der Gesetzgebung andererseits bislang auf dreierlei Weisen untersucht: Erstens liegen Arbeiten vor, die betrachten, ob das Abstimmungsverhalten einzelner Abgeordneter mit der Meinung der Wähler*innen im Wahlkreis korrespondiert (Miller und Stokes 1963; Achen 1978; Arnold 1990). Zweitens konnte mittels Daten des Comparative Policy Agenda Project zur themenspezifischen Gesetzgebung auf nationaler Ebene gezeigt werden, dass Verschiebungen in der öffentlichen Meinung durchaus Gesetzgebungsprozesse beeinflussen können (Jones und Baumgartner 2004; Froio et al. 2017). Drittens besteht die Möglichkeit, Responsivität auch breiter zu definieren und bereits die Be- 
fassung mit den Themen durch das Parlament als responsives Verhalten zu werten. Diese Strategie nutzt etwa Brettschneider (1995) in seiner wegweisenden Studie zu Deutschland.

Auf subnationaler Ebene wurde die Frage nach der Responsivität von Regierungen gegenüber den Präferenzen ihrer Bürger*innen bislang fast ausschließlich unter Rückgriff auf Daten für die US-Einzelstaaten untersucht - wobei hier neben Studien, die Staatsausgaben als abhängige Variable zur Messung der Policies verwenden (Pacheco 2013), auch Kodierungen gesetzgeberischer Outputs vorgenommen wurden (Lax und Phillips 2012). Für die deutschen Länder hingegen liegen weder Untersuchungen zur Responsivität vor, noch wurde die Gesetzgebung der Länder bisher themenspezifisch kodiert. Stattdessen nutzen die meisten Policy-Analysen im Bundesländervergleich - in der Tradition der Pionierstudie von Schmidt (1980) politikfeldspezifische Staatsausgaben (Wolf 2006; Hildebrandt 2009; Stocker 2010; Tepe und Vanhuysse 2014; Ewert et al. 2018) oder Personalstärken (z. B. Zahl der Lehrer oder Polizisten bei Tepe und Vanhuysse 2009, 2013) als abhängige Variable und streifen dabei die Frage der Responsivität allenfalls implizit, etwa bei der Untersuchung von strategischem Timing anhand von Wahlzyklen (Galli und Rossi 2002; Schneider 2009).

Anders verhält es sich mit Blick auf die vertikale Ebene, also hinsichtich der Frage nach der Beeinflussung von Entscheidungsfindung auf Länderebene durch den Bund. Hierzu besteht ein erheblicher Forschungsstand. Ein zentrales Thema ist dabei das Zusammenspiel zwischen Bund und Ländern im deutschen Föderalismus, wobei die These der Blockade effizienter Entscheidungsfindung im kooperativen Föderalismus der Bundesrepublik dominiert (Scharpf et al. 1976; Scharpf 1985; Lehmbruch 1976). Dabei entwickelte sich ein sehr ertragreicher Forschungsstrang, der sich insbesondere mit der Verflechtung von Entscheidungsfindungsprozessen zwischen Bund und Ländern und den daraus resultierenden Anpassungsproblemen an neue politische Herausforderungen beschäftigt (Benz und Lehmbruch 2002; Kropp 2010; Von Blumenthal und Bröchler 2010; Behnke und Kropp 2016).

Bei aller Ergiebigkeit dieser Forschungen fehlt jedoch bislang eine empirische Basis, mittels derer über eine systematische Erfassung der Gesetzgebung auf Länderebene, die Veränderungen von Politikverflechtung über Zeit quantifiziert und damit auch der Einfluss der Bundesebene analysiert werden könnte (aber mit Blick auf (De-)zentralisierungsdynamiken: Kaiser und Vogel 2017). Zwar haben mehrere Analysen zur Föderalismusreform I versucht, die Wirkungen der Reform zum Beispiel mit Blick auf die Zustimmungspflichtigkeit von Bundesgesetzen (Zohlnhöfer 2009; Stecker 2016) oder in Bezug auf die veränderten Kompetenzen der Länder (Stecker 2016; Reus und Vogel 2018) zu vermessen. Allerdings liegen für manche Bereiche (z. B. Abweichungsgesetzgebung) bisher keine systematischen Ergebnisse vor. Zudem leidet die bisherige Forschung darunter, dass eine längere Zeitreihe zur Gesetzgebungsaktivität in den Ländern in spezifischen Politikfeldern fehlt. Diese wäre jedoch notwendig, um unabhängig von den Wirkungen der jüngsten Großreform den Einfluss der Bundesebene zu analysieren. Denn die jahrzehntelange Forschung zu Zentralisierungs- und Dezentralisierungsdynamiken im deutschen Föderalstaat (Benz 1985; Biela et al. 2013; Kaiser und Vogel 2017) hat durchaus gezeigt, dass 
es dem Bund auch außerhalb großer Reformereignisse gelingt, zunehmend Einfluss auf die Landesgesetzgebung zu nehmen (siehe für einen Überblick Sturm 2016).

Aufgrund der fehlenden Datenbasis können die Erkenntnisse aus den vielfältigen Fallstudien bis dato noch nicht auf breiterer Basis abgesichert werden. Dieser Mangel an systematischen Daten zur Gesetzgebung erklärt auch, warum bisher nicht untersucht wurde, inwieweit die Kette der Responsivität nach Bund- und Landesebene spezifiziert werden kann. Für die Entwicklung von Sozialausgaben in den kanadischen Provinzen finden zum Beispiel Wlezien und Soroka (2011), dass die föderale Struktur Policy-Feedback massiv behindert und dadurch Responsivität im Sinne des thermostatic model auf nationaler Ebene eingeschränkt wird. Ein Grund hierfür liegt schlicht in der Verflechtung von Bund und Provinzen, die eine klare Zuschreibung von Verantwortung erschwert. Wenn solche Verflechtungen oder „Kontaminationen“ (Gschwend 2008) von politischen Prozessen stattfinden, hat dies direkte Auswirkungen auf die Kette der Responsivität, da zum Beispiel die Zuordnung von Verantwortlichkeit oder Regierungsperformanz verwischt wird (siehe auch Däubler et al. 2018).

Die reale Performanzwirkung politischen Handels (Policy-Outcomes) hängt dann von vielen Faktoren ab, die eine Landesregierung, trotz vielfältiger finanzieller und regulatorischer Instrumente nur teilweise beeinflussen kann, die ihr grundsätzlich zur Verfügung stehen. Neben dem Wollen spielt somit das Können einer Landesregierung im Hinblick auf die Umsetzung und Implementation getroffener Politikentscheidungen eine maßgebliche Rolle. Da ein responsiver Feedback-Zyklus idealiter nicht nur zwischen Policy-Outputs und Bürgerpräferenzen, sondern auch im Hinblick auf tatsächliche Policy-Outcomes bestehen sollte, kommt Studien wie der von Simonovits et al. (2019), die einen Zusammenhang zwischen ökonomischen Performanzindikatoren auf subnationaler Ebene und Wahlverhalten identifizieren können, eine große Bedeutung zu. Dabei kann allerdings auch eine große Diskrepanz zwischen tatsächlicher und von den Bürgern wahrgenommener Performanz bestehen.

Insgesamt lassen sich in den wenigen bisher vorhandenen Arbeiten zu Policy-Outcomes auf der Bundesländerebene relativ eindeutige Parteiendifferenzeffekte identifizieren (etwa Wurster und Hagemann 2018). Allerdings weist die Forschung hier auch auf zahlreiche weitere, zum Teil intervenierende oder Parteieneffekte verwischende ökonomische wie auch gesellschaftliche Einflussfaktoren hin. Responsive Politikgestaltung von Landesregierungen ihren Wählern gegenüber, stellt dabei, gerade auch wegen der Einflüsse des Mehrebenensystems, eine große Herausforderung dar. Trotz erheblicher methodischer Fallstricke ist die vergleichende Analyse der Policy-Outcomes für die Frage der Responsivität auf Bundesländereben allerdings ein weiterer entscheidender Baustein, da sie gleichsam den Endpunkt im politischen Prozess abbildet, und nur durch ihre Erfassung die gesamte Responsivitätskette samt etwaiger Feedbackschleife hinweg zu evaluieren ist.

\section{Skizze eines zukünftigen Forschungsprogramms}

Die Diskussion im vergangenen Abschnitt hat gezeigt, dass durchaus ein beeindruckender Forschungsstand zur Politik auf Bundesländerebene existiert. Gleichzeitig 
ist aber auch deutlich geworden, dass gerade aus der Perspektive der international-vergleichenden Forschung in wesentlichen Bereichen noch erhebliche Lücken bestehen, die es zu adressieren gilt, um eine Responsivitätskette auf Ebene der Bundesländer vollständig erfassen zu können. Wie könnte folglich ein zukünftiges Forschungsprogramm mit Blick auf die vergleichende Analyse von Politik und Policies auf Länderebene aussehen? Vor dem Hintergrund der vorangegangenen Erarbeitung des Forschungsstands sind unserer Ansicht nach vier ineinandergreifende Aspekte für eine Forschungsagenda besonders vielversprechend:

Erstens ist eine tiefergehende Auseinandersetzung mit einzelnen Elementen der Responsivitätskette mit Blick auf die Bundesländer notwendig, da hier teilweise noch (große) Lücken klaffen. So fehlen bisher etwa fundierte empirische Erkenntnisse nahezu vollständig, die es uns erlauben würden, gesicherte Aussagen über Prozesse der Problemdefinition auf Landesebene nachzuzeichnen. Für die Phase des Agenda-Settings und die Politikformulierung ist die Literaturlage zwar besser, weil die Programmatik der Landesparteien und deren Positionierung bereits vermessen wurden. Allerdings besteht noch erheblicher Forschungsbedarf bei der themenspezifischen Priorisierung sowie der Formulierung von spezifischen Politiken. Damit verbleibt die Untersuchung der Agendasetzung auf Ebene der Länder auf einem relativ hoch aggregierten Niveau und lässt direkte Rückschlüsse, inwiefern Parteien wichtige Themen der Bürger*innen in ihren Programmen adressieren, nicht zu. Für die Regierungspolitik und Politikimplementation kann wiederum festgehalten werden, dass es hier zwar eine große und wachsende Zahl von Studien zur BundLänder-Koordination oder zum Regierungshandeln in einzelnen Politikfeldern gibt, diese aber in der Regel keine systematisch-vergleichenden Einsichten über die Aktivität, Bilanz sowie Performanz von Regierungen auf Länderebene ermöglichen.

Zweitens fehlt eine systematische Betrachtung der Feedbackschleifen sowie der Rückkopplung der Ergebnisse in Bezug auf alle Elemente der Responsivitätskette. Neben der genaueren Betrachtung der Feedbackmechanismen als gesondertem Untersuchungsgegenstand erscheint es notwendig, im Sinne einer engeren Verschränkung der Kettenglieder, Feedbackelemente mit Blick auf alle Schritte der Responsivitätskette systematisch mitzudenken. So sind Feedbackelemente wie etwa Gesetze, die auf wahrgenommene Probleme zurückwirken, ebenso ein zentraler Bestandteil der Responsivitätskette wie die Wahrnehmung von Policy-Outcomes. Ohne ausreichende und systematisch erhobene empirische Daten beispielsweise darüber, welche Probleme in den Ländern als relevant betrachtet werden, lassen sich Aussagen über Rückwirkungseffekte von Gesetzen oder Policy-Outcomes allerdings nicht treffen.

Anknüpfend daran sollte die zukünftige Forschung drittens verstärkt die systematisch-empirische Analyse der Verbindungen zwischen den einzelnen Elementen in den Fokus ihrer Beschäftigung rücken und damit über die Fokussierung auf einzelne Elemente der Responsivitätskette hinauszielen. Stand heute liegen etwa nur wenige Erkenntnisse darüber vor, wie Einstellungen von Bürger*innen in den Ländern auf die Problemdefinition wirken oder welche Rolle andere bekannte Faktoren wie etwa fokussierende Ereignisse spielen. Darüber hinaus wissen wir nichts darüber, wie die Problemdefinition Motive und Zielsetzungen von Landesparteien bei der Formulierung von Wahlprogrammen beeinflusst. Auch tappen wir weitgehend im Dunkeln, wenn es um die Frage geht, wie identifizierte Probleme mittels Parteien 
als zentralen Transmissionsriemen in spezifische Politikpositionen und letztlich in Regierungshandeln übersetzt werden. Dabei wiegen die Forschungslücken in Bezug auf die einzelnen vorausliegenden Kettenglieder sowie die fehlende Analyse der Verknüpfungen der einzelnen Elemente besonders schwer für die systematische Untersuchung der Politikimplementation und Regierungsperformanz, die am Ende der Responsivitätskette steht bzw. im Sinne der Feedbackschleife wiederum auch ihren Anfang bildet. Wenn etwa unterschiedliche Gesetzgebung der Landesregierungen responsiv auf die Präferenzen der Wähler in den jeweiligen Bundesländern reagiert, dabei jedoch zu Divergenzen führt, stellt sich aus theoretischer Sicht die Frage, wie solche Entwicklungen föderalismustheoretisch und im Spannungsfeld von Einheit und Vielfalt im Bundesstaat zu interpretieren sind. Dabei gilt diese Problematik nicht nur für Analysen von Gesetzgebung und Performanz, sondern ist auch auf politische Einstellungen und Feedback übertragbar. Zwar lässt sich diese Frage je nach normativem föderalismustheoretischem Standpunkt unterschiedlich beantworten, mangels Daten zu den Ursachen unterschiedlicher Policy-Profile in den Ländern ist eine empirisch fundierte Diskussion solcher Zusammenhänge bisher jedoch kaum möglich.

Dies leitet über zum vierten zentralen Desiderat eines zukünftigen Forschungsprograms, nämlich der Schaffung einer einheitlichen empirischen Datengrundlage, welche umfassende(re) Analysen der Responsivitätskette - ihrer Einzelelemente sowie Verbindungen - als bisher ermöglicht. So ist ein wesentlicher Grund für viele der zuvor identifizierten Lücken darin zu suchen, dass eine empirische Untersuchung des gesamten Politikprozesses aufgrund mangelnder adäquater Daten bis dato schlichtweg nicht erfolgen konnte. Vor diesem Hintergrund sollte ein Ziel weiterer Forschungsaktivtäten sein, die Erhebung, Systematisierung und Bereitstellung von Daten auf Ebene der Bundesländer auszuweiten, beispielsweise zu den politischen Einstellungen der Bürger*innen in den Ländern, zur Themenaufmerksamkeit innerhalb der Bevölkerung, in regionalen Medien sowie in Parteiprogrammen, zu konkreten Politikpositionen von Parteien in Wahlprogrammen und Koalitionsverträgen, bis hin zur Parlaments- und Regierungspraxis sowie Performanzindikatoren.

Die in den vorausgegangenen Abschnitten aufgeworfenen Fragen zur Responsivitätskette sind insgesamt zu wichtig - sowohl mit Blick auf die Föderalismusforschung als auch mit Blick auf weitere demokratietheoretische Fragestellungen - um eine mögliche Varianz der Responsivität zwischen den Ländern, zwischen Politikfeldern sowie über Zeit nicht tiefergehend zu untersuchen. Daher stellt die systematische Aufarbeitung und empirische Analyse der skizzierten Responsivitätskette samt etwaiger Feedbackprozesse im Vergleich der deutschen Länder eine zentrale Forschungslücke dar, welche in Zukunft stärker adressiert werden sollte.

Danksagung Wir danken den Teilnehmern des Workshops „,Responsivitätsdynamiken im Spannungsfeld von Einheit und Vielfalt“ (IPW Heidelberg, November 2019) und insbesondere Marc Debus und Manfred G. Schmidt für hilfreiche Hinweise zu einer früheren Version dieses Aufsatzes.

Funding Open Access funding provided by Projekt DEAL.

Open Access Dieser Artikel wird unter der Creative Commons Namensnennung 4.0 International Lizenz veröffentlicht, welche die Nutzung, Vervielfältigung, Bearbeitung, Verbreitung und Wiedergabe in jeglichem Medium und Format erlaubt, sofern Sie den/die ursprünglichen Autor(en) und die Quelle ord- 
nungsgemäß nennen, einen Link zur Creative Commons Lizenz beifügen und angeben, ob Änderungen vorgenommen wurden.

Die in diesem Artikel enthaltenen Bilder und sonstiges Drittmaterial unterliegen ebenfalls der genannten Creative Commons Lizenz, sofern sich aus der Abbildungslegende nichts anderes ergibt. Sofern das betreffende Material nicht unter der genannten Creative Commons Lizenz steht und die betreffende Handlung nicht nach gesetzlichen Vorschriften erlaubt ist, ist für die oben aufgeführten Weiterverwendungen des Materials die Einwilligung des jeweiligen Rechteinhabers einzuholen.

Weitere Details zur Lizenz entnehmen Sie bitte der Lizenzinformation auf http://creativecommons.org/ licenses/by/4.0/deed.de.

\section{Literatur}

Achen, Christopher H. 1978. Measuring representation. American Journal of Political Science 22:475-510.

Adams, James F.Samuel Merrill, III, und Bernard Grofman. 2005. A unified theory of party competition: a cross-national analysis integrating spatial and behavioral factors. Cambridge: Cambridge University Press.

Alonso, Sonia, Braulio Gómez, und Laura Cabeza 2013. Measuring centre-periphery preferences: the regional manifestos project. Regional \& Federal Studies 23:189-211.

Arnold, R. Douglas. 1990. The logic of congressional action. New Haven: Yale University Press.

Bauer, Michael W., und Christoph Knill. 2014. A conceptual framework for the analysis of policy change: measurement, explanation and strategies of policy dismantling. Journal of Comparative Policy Analysis 16:28-44.

Baumann, Markus, Marc Debus, und Martin Gross. 2017. Strength of weakness? Innerparteiliche Heterogenität, divergierende Koalitionspräferenzen und die Ergebnisse von Koalitionsverhandlungen in den deutschen Bundesländern. Politische Vierteljahresschrift 58:179-204.

Baumgartner, Frank R., und Bryan D. Jones. 1991. Agenda dynamics and policy subsystems. The Journal of Politics 53(4):1044-1074.

Baumgartner, Frank R., Christoffer Green-Pedersen, und Bryan D. Jones. 2006. Comparative studies of policy agendas. Journal of European Public Policy 13:959-974.

Behnke, Nathalie, und Sabine Kropp. 2016. Arraying institutional layers in federalism reforms: lessons from the German case. Regional \& Federal Studies 26:585-602.

Benoit, Kenneth, und Michael Laver. 2006. Party policy in modern democracies. London: Routledge.

Benz, Arthur. 1985. Föderalismus als dynamisches System. Zentralisierung und Dezentralisierung im föderativen Staat. Beiträge zur sozialwissenschaftlichen Forschung. Opladen: Westdeutscher Verlag.

Benz, Arthur, und Gerhard Lehmbruch. 2002. Föderalismus - Analysen in entwicklungsgeschichtlicher und vergleichender Perspektive. Wiesbaden: Westdeutscher Verlag.

Best, Volker. 2015. Koalitionssignale bei Landtagswahlen: Eine empirische Analyse von 1990 bis 2012. Baden-Baden: Nomos.

Biela, Jan, Annika Hennl, und André Kaiser. 2013. Policy making in multilevel systems. Federalism, Decentralisation, and performance in the OECD countries. Colchester: ECPR Press.

von Blumenthal, Julia. 2010. Im Zweifel für die Einheit(lichkeit)? Determinanten landespolitischer Entscheidungen. In Föderalismusreform in Deutschland: Bilanz und Perspektiven im internationalen Vergleich, Hrsg. Julia von Blumenthal, Stephan Bröchler, 177-196. Wiesbaden: VS.

von Blumenthal, Julia, und Stephan Bröchler. 2010. Föderalismusreform in Deutschland: Bilanz und Perspektiven im internationalen Vergleich. Wiesbaden: VS.

Bolleyer, Nicole. 2014. Sub-national politics: a comparative perspective. In The oxford handbook of state and local government, Hrsg. Donald P. Haider-Markel, 888-912. New York: Oxford University Press.

Bräuninger, Thomas, und Marc Debus. 2012. Parteienwettbewerb in den deutschen Bundesländern. Wiesbaden: Springer.

Bräuninger, Thomas, und Nathalie Giger. 2018. Strategic ambiguity of party positions in multi-party competition. Political Science Research Methods 6(3):527-548. https://doi.org/10.1017/psrm.2016.18.

Bräuninger, Thomas, Marc Debus, Jochen Müller, und Christian Stecker. 2019. Party competition and government formation in Germany: business as usual or new patterns? German Politics 28:80-100.

Bräuninger, Thomas, Marc Debus, Jochen Müller und Christian Stecker. 2020. Parteienwettbewerb in den deutschen Bundesländern. Wiesbaden: Springer VS. 
Brettschneider, Frank. 1995. Öffentliche Meinung und Politik. Eine empirische Studie zur Responsivität des deutschen Bundestags zwischen 1949 und 1990. Opladen: Westdeutscher Verlag.

Budge, Ian. 2015. Issue emphases, saliency theory and issue ownership: a historical and conceptual analysis. West European Politics 38:761-777.

Cabeza, Laura. 2018. 'First-order thinking' in second-order contests: A comparison of local, regional and European elections in Spain. Electoral Studies 53:29-38.

Cabeza, Laura, Braulio Gómez, und Sonia Alonso. 2016. How national parties nationalize regional elections: the case of Spain. Publius: The Journal of Federalism 47:77-98.

Caughey, Devin, und Christopher Warshaw. 2017. Policy preferences and policy change: dynamic responsiveness in the American states, 1936-2014. American Political Science Review 112:249-266.

Clasen, Jochen, und Nico A. Siegel. 2007. Investigating welfare state change: the 'dependent variable problem' in comparative analysis. Cheltenham: Edward Elgar.

Dalton, Russell J., David M. Farrell und Ian McAllister. 2011. Political parties and democratic linkage: how parties organize democracy. Oxford: Oxford University Press.

Däubler, T., J. Müller, und C. Stecker. 2018. Assessing democratic representation in multi-level democracies. West European Politics 41:541-564.

Debus, Marc. 2017. Die Thematisierung der Flüchtlingskrise im Vorfeld der Landtagswahlen 2016: Mangelnde Responsivität als eine Ursache für den Erfolg der AfD? In Regieren in der Einwanderungsgesellschaft: Impulse zur Integrationsdebatte aus Sicht der Regierungsforschung, Hrsg. Christoph Bieber, Andreas Blätte, Karl-Rudolf Korte, und Nico Switek, 91-98. Wiesbaden: Springer.

Esaiasson, Peter, und Christopher Wlezien. 2017. Advances in the study of democratic responsiveness: an introduction. Comparative Political Studies 50:699-710.

Ewert, Stefan, Michael Jankowski, und Jochen Müller. 2018. Politikpositionen von Regierungen im Ländervergleich. Zeitschrift für Vergleichende Politikwissenschaft 12:683-702.

Freitag, Markus, und Adrian Vatter. 2008. Die Demokratien der deutschen Bundesländer. Opladen: Barbara Budrich (UTB).

Froio, Caterina, Shaun Bevan, und Will Jennings. 2017. Party mandates and the politics of attention: party platforms, public priorities and the policy agenda in britain. Party Politics 23(6):692-703.

Galli, Emma, und Rossi P.S. Stefania. 2002. Political budget cycles: the case of the western German Länder. Public Choice 110:283-303.

Golder, Sona N., Ignacio Lago, André Blais, Elisabeth Gidengil, et al. 2017. Multi-level electoral politics. Oxford: Oxford University Press.

Green-Pedersen, Christoffer. 2004. The dependent variable problem within the study of welfare state retrenchment: defining the problem and looking for solutions. Journal of Comparative Policy Analysis 6:3-14.

Gross, Martin, und Michael Jankowski. 2019. Dimensions of political conflict and party positions in multilevel democracies: evidence from the Local Manifesto Project. West European Politics https://doi. org/10.1080/01402382.2019.1602816.

Gschwend, Thomas. 2008. Studying contamination effects in multi-level systems of governance: first thoughts about hypotheses and research design. In The multilevel system of the EU, Hrsg. Cees van der Eijk, Hermann Schmitt, 229-240. Mannheim: CONNEX Report Series.

Hegele, Yvonne, und Nathalie Behnke. 2013. Die Landesministerkonferenzen und der Bund - Kooperativer Föderalismus im Schatten der Politikverflechtung. Politische Vierteljahresschrift 54:21-49.

Hildebrandt, Achim. 2009. Die finanzpolitische Handlungsfähigkeit der Bundesländer: Determinanten, institutionelle Defizite und Reformoptionen. Wiesbaden: VS.

Hildebrandt, Achim, und Frieder Wolf. 2008. Die Politik der Bundesländer. Staatstätigkeit im Vergleich. Wiesbaden: VS.

Hildebrandt, Achim, und Frieder Wolf. 2016. Die Politik der Bundesländer: Zwischen Föderalismusreform und Schuldenbremse. Wiesbaden: Springer VS.

Hörisch, Felix, und Stefan Wurster. 2017. Das grün-rote Experiment in Baden-Württemberg. Eine Bilanz. der Landesregierung Kretschmann 2011-2016. Wiesbaden: Springer VS.

Hörisch, Felix, und Stefan Wurster. 2019. The policies of the first green-red government in the German federal state of Baden-Württemberg, 2011-2016. A fuzzy-set qualitative comparative analysis of different policy sectors. Politische Vierteljahresschrift 60(3):513-538.

Jeffery, Charlie, Niccole M. Pamphilis, Carolyn Rowe, und Ed Turner. 2016. Introduction to the special issue: reframing German federalism. German Politics 25:165-175.

Jensen, Carsten, und Georg Wenzelburger. 2020. Reforming the welfare state. London: Routledge.

Jones, Bryan D. 1994. Reconceiving decision-making in democratic politics. Chicago: University of Chicago Press. 
Jones, Bryan D., und Frank R. Baumgartner. 2004. Representation and agenda setting. Policy Studies Journal 32:1-24.

Jun, Uwe, Melanie Haas, und Oskar Niedermayer. 2008. Parteien und Parteiensysteme in den deutschen Ländern. Wiesbaden: VS.

Kaiser, André, und Stephan Vogel. 2017. Dynamic De/Centralization in Germany, 1949-2010. Publius: The Journal of Federalism 49(1):84-111.

Kingdon, Jonathan. 1984. Agendas, alternatives, and public policies. Boston: Little, Brown.

Klingemann, Hans-Dieter, Richard I. Hofferbert, und Ian Budge. 1994. Parties, policies, and democracy. Boulder, San Francisco, Oxford: Westview.

Kortmann, Matthias, und Christian Stecker. 2019. Party competition and immigration and integration policies: a comparative analysis. Comparative European Politics 17(1):72-91.

Kropp, Sabine. 2010. Kooperativer Föderalismus und Politikverflechtung. Wiesbaden: VS.

Krumpal, Ivar, und Heiko Rauhut. 2006. Dominieren Bundes- oder Landesparteien die individuellen Landtagswahlentscheidungen in der BRD? Eine quantitative Analyse zum Ausmaß der bundespolitischen Parteipolitikverflechtung bei Landtagswahlen (1996-2000). Arbeitsbericht des Instituts für Soziologie, Nr. 45.

Laver, Michael, und William B. Hunt. 1992. Policy and party competition. New York: Routledge.

Lax, Jeffrey R., und Justin H. Phillips. 2012. The democratic deficit in the states. American Journal of Political Science 56:148-166.

Lehmbruch, Gerhard. 1976. Parteienwettbewerb im Bundesstaat. Stuttgart: Kohlhammer.

Leunig, Sven. 2007. Die Regierungssysteme der deutschen Länder im Vergleich. UTB Politikwissenschaft, Bd. 2844. Opladen: Budrich.

Miller, Warren E., und Donald E. Stokes. 1963. Constituency influence in congress. American Political Science Review 57:45-56.

Müller, Jochen. 2013. On a short leash? Sub-national party positions between regional context and national party unity. Journal of Elections, Public Opinion and Parties 23:177-199.

Müller, Jochen, und Marc Debus. 2012. „Second-order“-Effekte und Determinanten der individuellen Wahlentscheidung bei Landtagswahlen: Eine Analyse des Wahlverhaltens im deutschen Mehrebenensystem. Zeitschrift für Vergleichende Politikwissenschaft 6:17-47.

Pacheco, Julianna. 2013. The thermostatic model of responsiveness in the American states. State Politics \& Policy Quarterly 13:306-332.

Page, Benjamin I., Robert Y. Shapiro, (1983) Effects of Public Opinion on Policy. American Political Science Review 77 (1):175-190.

Pitkin, Hanna. 1967. The Concept of Representation. Berkeley: University of California Press.

Polk, Jonathan, Jan Rovny, Ryan Bakker, Erica Edwards, Liesbet Hooghe, Seth Jolly, et al, 2017. Explaining the salience of anti-elitism and reducing political corruption for political parties in Europe with the 2014 Chapel Hill Expert Survey data. Research \& Politics 4:2053168016686915.

Powell, G. Bingham. 2004. The chain of responsiveness. Journal of Democracy 15:91-105.

Przeworski, Adam, Susan C. Stokes, und Bernard Manin. 1999. Democracy, accountability, and representation. Cambridge: Cambridge University Press.

Rauh, Christina A. 2016. Wahlkampf im Kontext.Negative Kampagnenstrategien in 58 Landtagswahlen. Wiesbaden: Springer VS.

Reus, Iris, und Stephan Vogel. 2018. Policy-Vielfalt zwischen den Bundesländern nach der Föderalismusreform I: Art, Ausmaß und Akteure. Zeitschrift für Vergleichende Politikwissenschaft 12:621-642.

Sack, Detlef, und Annette Elisabeth Töller. 2018. Einleitung: Policies in den deutschen Ländern. Zeitschrift für Vergleichende Politikwissenschaft 12:603-619.

Scharpf, Fritz W. 1985. Die Politikverflechtungs-Falle: Europäische Integration und deutscher Föderalismus im Vergleich. Politische Vierteljahresschrift 26:323-356.

Scharpf, Fritz W., Bernd Reissert, und Fritz Schnabel. 1976. Politikverflechtung. Theorie und Empirie des kooperativen Föderalismus in der Bundesrepublik. Kronberg: Cornelsen Scriptor.

Schmidt, Manfred G. 1980. CDU und SPD an der Regierung. Ein Vergleich ihrer Politik in den Ländern. Frankfurt am Main, New York: Campus.

Schneider, Christina J. 2009. Fighting with one hand tied behind the back: political budget cycles in the West German states. Public Choice 142:125.

Seher, Nicole M., und Franz Pappi. 2011. Politikfeldspezifische Positionen der Landesverbände der deutschen Parteien. MZES Working Paper 139. Mannheim: Mannheimer Zentrum für Europäische Sozialforschung.

Simonovits, Gabor, Andrew M. Guess, und Jonathan Nagler. 2019. Responsiveness without representation: evidence from minimum wage laws in U.S. States. American Journal of Political Science 63:401-410. 
Soroka, Stuart N., und Christopher Wlezien. 2010. Degrees of democracy: politics, public opinion, and policy. Cambridge: Cambridge University Press.

Soroka, Stuart N., und Christopher Wlezien. 2015. The majoritarian and proportional visions and democratic responsiveness. Electoral Studies 40:539-547.

Spier, Tim. 2013. Große Koalitionen in den deutschen Bundesländern 1949-2013. ZPol Zeitschrift für Politikwissenschaft 23:489-516.

Stecker, Christian. 2015. Parties on the chain of federalism: position-taking and multi-level party competition in Germany. West European Politics 38:1305-1326.

Stecker, Christian. 2016. The effects of federalism reform on the legislative process in Germany. Regional \& Federal Studies 26:603-624.

Stocker, Rainer. 2010. Die Kulturpolitik der deutschen Bundesländer im Vergleich. In Vergleichende subnationale Analysen für Deutschland. Institutionen, Staatstätigkeiten und politische Kulturen, Hrsg. Markus Freitag, Adrian Vatter, 203-233. Münster: LIT.

Sturm, Roland. 2016. Autonomieverlust der Länder. Föderale Kompetenzverteilung in Deutschland. In Föderale Kompetenzverteilung in Europa, Hrsg. Anna Gamper, Peter Bußjäger, Ferdinand Karlhofer, Günther Pallaver, und Walter Obwexer, 129-150. Baden-Baden: Nomos.

Tenscher, Jens, und Uta Rußmann. 2016. Vergleichende Wahlkampfforschung: Studien anlässlich der Bundestags- und Europawahlen 2013 und 2014. Wiesbaden: Springer VS.

Tenscher, Jens, und Sonja Schmid. 2009. Berichterstattung nach Wahl. Eine vergleichende Analyse von Bundes- und Landtagswahlkämpfen in der Regionalpresse. Medien \& Kommunikationswissenschaft 57:56-77.

Tepe, Markus, und Pieter Vanhuysse. 2009. Educational business cycles. Public Choice 139:61-82.

Tepe, Markus, und Pieter Vanhuysse. 2013. Cops for hire? The political economy of police employment in the German states. Journal of Public Policy 33:165-199.

Tepe, Markus, und Pieter Vanhuysse. 2014. A vote at the opera? The political economy of public theaters and orchestras in the German states. European Journal of Political Economy 36:254-273.

Volkens, Andrea, Werner Krause, Pola Lehmann, Theres Matthieß, Nicolas Merz, Sven Regel, et al. 2018. The manifesto data collection. Manifesto project (MRG/CMP/MARPOR). Berlin: Wissenschaftszentrum für Sozialforschung (WZB). Version b.

Völkl, Kerstin. 2008. Reine Landtagswahlen oder regionale Bundestagswahlen? Eine Untersuchung des Abstimmungsverhaltens bei Landtagswahlen 1990-2006. Baden-Baden: Nomos.

Völkl, Kerstin, Kai-Uwe Schnapp, Everhard Holtmann, und Oscar W. Gabriel (Hrsg.). 2008. Wähler und Landtagswahlen in der Bundesrepublik Deutschland. Baden-Baden: Nomos.

Wagschal, Uwe, und Georg Wenzelburger. 2009. Determinanten der Haushaltskonsolidierung der Bundesländer. Zeitschrift für Vergleichende Politikwissenschaft 3(1):33-58.

Wenzelburger, Georg. 2013. Das goldene Zeitalter der Vermögenserlöse. Die Determinanten der Einmalerlöse der Bundesländer 1995-2007. Politische Vierteljahresschrift 54:111-154.

Wenzelburger, Georg. 2020. The partisan politics of law and order. Oxford: Oxford University Press.

Wenzelburger, Georg, Carsten Jensen, Lee Seonghui, und Christoph Arndt. 2019. How governments strategically time welfare state reform legislation: empirical evidence from five European countries. West European Politics https://doi.org/10.1080/01402382.2019.1668245.

Wlezien, Christopher. 2016. Public opinion and policy representation: on conceptualization, measurement, and interpretation. Policy Studies Journal https://doi.org/10.1111/psj.12190.

Wlezien, Christopher, und Stuart N. Soroka. 2011. Federalism and public responsiveness to policy. Publius: The Journal of Federalism 41:31-52.

Wlezien, Christopher, und Stuart N.S.N. Soroka. 2012. Political institutions and the opinion-policy link. West European Politics 35:1407-1432.

Wolf, Frieder. 2006. Die Bildungsausgaben der Bundesländer im Vergleich. Münster: LIT.

Wurster, Stefan. 2017. Energiewende in Baden-Württemberg: Ausmaß und Folgen. In Das grün-rote Experiment. Eine Bilanz der Landesregierung Kretschmann 2011-2016, Hrsg. Felix Hörisch, Stefan Wurster, 251-278. Wiesbaden: Springer VS.

Wurster, Stefan, und Christian Hagemann. 2018. Two ways to success-Expansion of renewable energies in comparison between Germany's federal states. Energy Policy 119:610-619.

Wurster, Stefan, und Christian Hagemann. 2019. The expansion of renewable energy in federal settings: Austria, Belgium and Germany in comparison. Journal of Environment and Development 29(1):147-168. https://doi.org/10.1177/1070496519887488.

Zohlnhöfer, Reimut. 2009. Der Politikverflechtungsfalle entwischt? Die Effekte der Föderalismusreform I auf die Gesetzgebung. Zeitschrift für Politikwissenschaft 19:39-76. 